\title{
Searching for gravitational waves from the Crab pulsar - the problem of timing noise
}

\author{
Matthew Pitkin and Graham Woan \\ Department of Physics and Astronomy, Kelvin Building, University of Glasgow, \\ Glasgow G12 8QQ, UK \\ E-mail: matthew@astro.gla.ac.uk
}

\begin{abstract}
Of the current known pulsars, the Crab pulsar $($ B0531+21) is one of the most promising sources of gravitational waves. The relatively large timing noise of the Crab causes its phase evolution to depart from a simple spin-down model. This effect needs to be taken in to account when performing time domain searches for the Crab pulsar in order to avoid severely degrading the search efficiency. The Jodrell Bank Crab pulsar ephemeris is examined to see if it can be used for tracking the phase evolution of any gravitational wave signal from the pulsar, and we present a method of heterodyning the data that takes account of the phase wander. The possibility of obtaining physical information about the pulsar from comparisons of the electromagnetically and a gravitationally observed timing noise is discussed. Finally, additional problems caused by pulsar glitches are discussed.
\end{abstract}




\section{Introduction}

Of the known isolated pulsars the Crab pulsar $(\mathrm{B} 0531+21)$ is thought to be one of the most promising sources of gravitational waves. This is due to its relative youth ( 1000 years) and correspondingly large spin-down rate. The criterion which makes the Crab pulsar such a promising source also provides one of the major problems faced for search algorithms designed to detect it, that of timing noise. Timing noise has been known about since the early days of pulsar observations, and represents a random walk in phase or frequency of the pulsar about the regular spin-down model (Cordes and Helfand, 1980). It is thought to be intrinsic to the pulsar and not just a result of magnetospheric perturbations or interstellar propagation. Several different models for its origin have been suggested (Cordes and Greenstein, 1981) although its origin is still unknown. Timing noise has been shown to be well correlated with spin-down rate (Arzoumanian et al , 1994), and therefore it is no surprise that the Crab pulsar has a large timing noise component. Many other pulsars are seen to have timing noise, but it is negligible for almost all of those within the frequency band of the LIGO and GEO600 interferometers.

\section{The Crab pulsar ephemeris}

The Crab pulsar has been extensively studied since 1969 and its behaviour from 15 February 1982 to the present is recorded in a monthly ephemeris produced at Jodrell Bank (http://www.jb.man.ac.uk/pulsars/crab.htmi). This contains the pulse arrival time at the solar system barycentre (given as the arrival time of the peak of the first pulse after midnight on the given day), the frequency $(\nu)$ and first frequency derivative $(\dot{\nu})$ of the pulsar. The phase residuals caused by timing noise can be seen in the Jodrell Bank ephemeris after removing the third order Taylor expansion of the phase,

$$
\phi=\phi_{0}+\nu_{0}\left(t-t_{0}\right)+\frac{1}{2} \dot{\nu_{0}}\left(t-t_{0}\right)^{2}+\frac{1}{6} \ddot{\nu_{0}}\left(t-t_{0}\right)^{3},
$$

from that calculated using the ephemeris data (see figure 1). There is a good agreement between figure 1 and phase residual plots in figure 4 of Lyne et al (1993) and Scott et al (2003) indicating that the ephemeris is sufficient to track the Crab pulsar phase. Any difference between figure 1 and those in the above references is most probably due to different lengths of data used for the fitting procedure. Lyne et al (1993) showed that the timing noise does not vary significantly on scales of less than a month, meaning that the ephemeris should have sufficient time resolution.

\section{Search method}

Current search methods for detecting gravitational waves from known pulsars, as developed for the LIGO and GEO600 analyses (The LIGO Scientific Collaboration 2003), require an accurate knowledge of their phase evolution, and any timing noise 


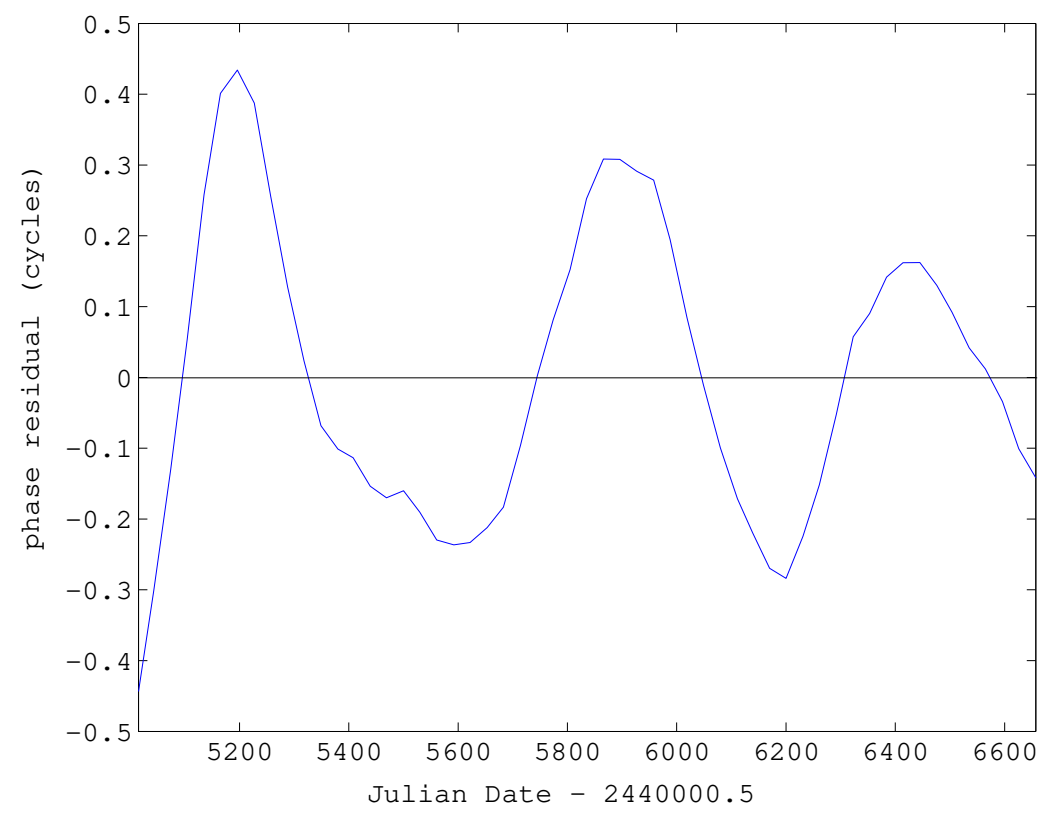

Figure 1. The effect of timing noise as seen in the phase residual of the Crab pulsar obtained by removing a third order fit from the phase as calculated using the Jodrell Bank Crab pulsar ephemeris.

has to be correctly accounted for. The gravitational wave signal from a pulsar as seen at the detector (Jaranowski et al, 1998) is defined as

$$
h(t)=F_{+}(t, \psi) h_{0} \frac{1+\cos ^{2} \iota}{2} \cos \Phi(t)+F_{\times}(t, \psi) h_{0} \cos \iota \sin \Phi(t),
$$

where $F_{+}$and $F_{\times}$are the detector's beam pattern dependent on the gravitational wave polarisation angle $\psi, \iota$ is the angle between the pulsar's spin direction and the propagation direction of the gravitational waves and $\Phi(t)$ is the phase evolution of the pulsar including Doppler effects from the Earth's rotation and orbital motion. The time domain search method developed by Dupuis and Woan (in preparation) and used in LIGO Scientific Collaboration (2003) uses heterodyning procedures to remove the time varying parts of $\Phi$ (as determined through radio observations). This leaves a gravitational wave signal that would vary only with the detector beam patterns. The analysis method uses Bayesian inference to infer posterior probability densities for the unknown pulsar parameters of $h_{0}, \iota, \psi$ and $\phi_{0}$ (the pulsar's phase at reference time $t_{0}$ ). This method is fine for pulsars with small timing noise, and would be fine for the Crab pulsar over short integration periods of days, but as signals from pulsars will be weak very long integration times of months to years are needed. The timing noise in the Crab pulsar will cause the phase to drift significantly from a simple spin-down model over a relatively short time, thus degrading the efficiency of or completely nullifying the search method. Therefore the timing noise has to be dealt with by applying an extra heterodyne that removes its effects. Here the ephemeris data needs to be used to track 
the phase. The complete phase evolution can be calculated by applying a spline fit between consecutive monthly frequencies in the ephemeris using the frequency, its first derivative and the phase as boundary conditions. This produces a fifth order equation for the phase of the form

$$
\phi=\phi_{0}+\nu_{0}\left(t-t_{0}\right)+\frac{1}{2} \dot{\nu_{0}}\left(t-t_{0}\right)^{2}+\frac{1}{6} \ddot{\nu_{0}}\left(t-t_{0}\right)^{3}+\frac{1}{24} \ddot{\nu_{0}}\left(t-t_{0}\right)^{4}+\frac{1}{120} \ddot{\nu_{0}}\left(t-t_{0}\right)^{5},
$$

in which the spin-down parameters $\ddot{\nu_{0}}, \ddot{\nu_{0}}$ and $\ddot{\nu_{0}}$ vary on a monthly basis. The phase as calculated in equation (3) is then removed in the extra heterodyning stage of the analysis,

$$
\text { heterodyned data }=\text { data } \times \exp \left(-\phi_{\text {timing noise }}\right) \text {. }
$$

It is acknowledged that this interpolation method is maybe not the most accurate fitting technique although it seems adequate for our purposes.

\section{Discussion and conclusions}

Timing noise may not just be a nuisance that gets in the way of our search method, but may provide some astrophysical insight into the structure of neutron stars. It is generally assumed that the neutron star and its magnetosphere are strongly coupled. Any difference in the timing noise phases between gravitational and electromagnetic radiation will provide information on the strength of this coupling. To test this coupling a new parameter, $\alpha$, can be added into the search algorithm of Dupuis and Woan. This parameter has been investigated by Jones (private communication) and is defined as the scaling factor between the electromagnetic and gravitational phase noise, reflecting changes in the moment of inertia and magnetosphere of the neutron star. This information would be useful in constraining the various equation of state models of neutron stars.

As well as timing noise the Crab pulsar, like many other pulsars, is also subject to glitches. These are characterised by a sudden increase in the pulsar frequency and spin-down rate, followed by a slower decay back to close to their pre-glitch values. These glitches also need to be taken into account in searches, in order to avoid the uncertain phase evolution around them decreasing the search efficiency. It is unknown what effect glitches could have on the gravitational wave phase, due to possible changes in the moment of inertia or crustal structure of the pulsar, so that after each glitch a new initial phase parameter will be needed in the search algorithm to take into account a possible jump in phase. This is equivalent to performing coherent searches only between glitches, and then combining the results incoherently.

The Crab pulsar is not unique in its large timing noise or glitchiness, and as gravitational wave detectors develop better sensitivity at lower wavelengths the methods highlighted above will need to be further developed and applied more often in pulsar analyses. This in turn will require close observation in radio wavelengths to characterise the phase evolution. In cases where radio observations are not regularly available 
comparisons should be made of different methods of fitting to the data that provide the best extrapolation results. It is also worthwhile to study the effects of timing noise and how it effects the search efficiency and coherence times when thinking about searches for unknown pulsars, the most promising candidates for which are young and therefore many are likely to have large timing noise. We are requesting additional Crab timing data from Jodrell Bank on a shorter timescale with which to test the spline interpolation and give a more precise tracking of the phase.

The prospect of getting physical information about pulsars from the comparisons of electromagnetic and gravitational timing noise makes these considerations particularly important.

\section{References}

Arzoumanian Z et al 1994 Ap. J. 422 671-680

Cordes J M and Helfand D J 1980 Ap. J. 239 640-650

Cordes J M and Greenstein G 1981 Ap. J. 245 1060-1079

Dupuis $\mathrm{R}$ and Woan $\mathrm{G}$ in preparation

Jaranowski P et al 1998 Phys. Rev. D 5863001

Jones D I private communication

Jodrell Bank Crab Pulsar Timing: Monthly Ephemeris http://www.jb.man.ac.uk/research/pulsar/crab.html

Lyne A et al 1993 Mon. Not. R. Astron. Soc. 265 1003-1012

Scott D M et al 2003 Mon. Not. R. Astron. Soc. 344 412-430

The LIGO Scientific Collaboration; Abbott B et al 2003 Setting upper limits on the strength of periodic gravitational waves using the first science data from the GEO600 and LIGO detectors Preprint gr-qc/0308050 